\title{
Mitral valve motion after performing an edge-to-edge repair in an isolated swine heart
}

\author{
Hiroki Hasegawa, MD, Yoshimori Araki, MD, PhD, Akihiko Usui, MD, PhD, Jun Yokote, MD, Shunei Saito, MD, PhD,
} Hideki Oshima, MD, PhD, and Yuichi Ueda, MD, PhD

Video clip is available online.
From the Department of Cardiothoracic Surgery, Nagoya University Graduate School of Medicine, Nagoya, Japan.

Received for publication Nov 21, 2007; revisions received Feb 2, 2008; accepted for publication March 7, 2008.

Address for reprints: Hiroki Hasegawa, MD, Department of Cardiothoracic Surgery, Nagoya University Graduate School of Medicine, 65 Tsurumai-cho, Showa-ku, Nagoya, Aichi, 466-8550, Japan (E-mail: hirokih@ med.nagoya-u.ac.jp).

J Thorac Cardiovasc Surg 2008;136:590-6 0022-5223/\$34.00

Copyright $(\underset{2008}{ } 20 y$ The American Association for Thoracic Surgery

doi:10.1016/j.jtcvs.2008.03.050
Objectives: Mitral valve motion after performing an edge-to-edge repair is a major concern, but it has not yet been observed directly. Mitral valve motion was assessed by using a high-speed digital video camera and microsonometric analysis in an isolated swine heart, and any changes in the mitral valve area or the development of mitral stenosis symptoms were evaluated.

Methods: A temporary edge-to-edge repair model was created. The mitral valve motion was assessed by using a high-speed digital video camera, and the motion of the mitral annulus was measured by means of sonomicrometric analysis with or without performing edge-to-edge repair $(n=5)$. The left ventricular volumetric measurements were also measured with a conductance catheter. One cardiac cycle was divided into 4 phases: the mitral valve open phase, the isovolumic contraction phase, the aortic valve open phase, and the isovolumic relaxation phase.

Results: The mitral valve was divided into 2 orifices by using the edge-to-edge technique, and the mitral valve area decreased by approximately $30 \%$. The ratio of mitral valve open phase significantly decreased $(31.9 \% \pm 3.4 \%$ vs $41.4 \% \pm 3.7 \%, P=.04)$. There were no significant differences in the diameter and the changes of anteroposterior dimensions of the mitral annulus. The stroke volume and the peak positive and negative $\mathrm{dp} / \mathrm{dt}$ values showed no obvious change, but dv/dt values increased slightly without significance after removing the edge-to-edge suture $(118 \pm 25$ vs $130 \pm 17$ $\mathrm{mL} / \mathrm{s}, P=.14)$.

Conclusions: The mitral valve area decreased slightly; however, edge-to-edge repair did not create symptomatic mitral stenosis and showed no adverse affects on cardiac function.

$\mathrm{E}$ dge-to-edge (EtoE) repair technique was first introduced by Fucci and colleagues $^{1}$ in 1995 as a simple method of mitral valve plasty. It has since been performed in patients with mitral valve prolapse because of its technical simplicity. ${ }^{2,3}$ Satisfactory early and midterm results have been reported for this technique. ${ }^{4-6}$

There is concern that the mitral valve area (MVA) might be reduced after performing EtoE repair because of the double orifices, and symptoms of mitral stenosis (MS) can develop. Functional MS after performing EtoE repair has not yet been described in previous reports. In those reports the MVA was evaluated by means of echocardiographic analysis. ${ }^{4,5,7,8}$

Mitral valve motion after EtoE repair has been discussed; however, there have been no direct observations or evaluations of mitral valve motion after EtoE repair. The changes in mitral valve status, including leaflet motion and the timing of opening and closing, should therefore be clarified.

Our group developed an isolated and working swine heart model using crystalloid perfusate to examine the valve motion with a high-speed digital video camera system 

Abbreviations and Acronyms
EtoE = edge-to-edge
LAP $=$ left atrial pressure
$\mathrm{LV}=$ left ventricular
MS = mitral stenosis
MVA $=$ mitral valve area
MVOP $=$ mitral valve open phase
$\mathrm{NM}=$ normal mode
$\mathrm{SV}=$ stroke volume

in $2005 .^{9,10}$ This model enables the direct observation or examination of mitral valve motion in a working heart.

The present study was designed to clarify the motion of mitral valve leaflets and annulus before and after performing EtoE repair and to detect any changes in the MVA or the development of MS symptoms as well.

\section{Materials and Methods \\ Temporary EtoE Model}

A temporary EtoE model was created to observe the motion of the mitral valve leaflets and annulus before and after EtoE repair. A temporary EtoE suture was created with a U stitch by using a 5-0 polypropylene suture, which was placed in the middle portion of the leaflet edge. The suture was tied in a bow, and a 3-0 silk suture was passed through one loop of the bow knot. Every suture was passed through the left atrial wall. When the silk was pulled out on beating, the polypropylene suture was pulled out, the EtoE knot came untied, and the normal mitral valve was restored (Figure 1). This status is called the normal mode (NM).

\section{Study Protocol}

All measurements were obtained in a swine isolated and working heart model perfused with crystalloid perfusate. The following pressure study was performed in all 10 cases: aortic pressure, left ventricular (LV) pressure, left atrial pressure (LAP), and results of electrocardiographic analysis were measured and recorded.

The motion of the mitral valve leaflets and annulus was evaluated in 5 swine hearts (protocol 1). After the pressure study, a high-speed digital video camera was introduced to the left ventricle through the apex. The mitral valve motion was directly observed with this camera, and the anteroposterior dimension of mitral annular diameter was consecutively measured by means of sonomicrometric analysis. All measurements were performed on a temporary EtoE model first, and then the EtoE suture was released. The normal mitral valve was restored, and the motion of normal mitral valve leaflets and annulus was observed. These results were compared before and after removing the EtoE suture.

In protocol 2 the $\mathrm{LV}$ volumetric measurements were carried out by using a conductance catheter in the other 5 swine hearts. The measurements were performed before and after removing the EtoE suture to evaluate the LV filling pattern and any adverse affects of the EtoE repair on the MVA.

The research protocol was approved by the Nagoya University Laboratory Research Animal Care and Ethics Committee. The

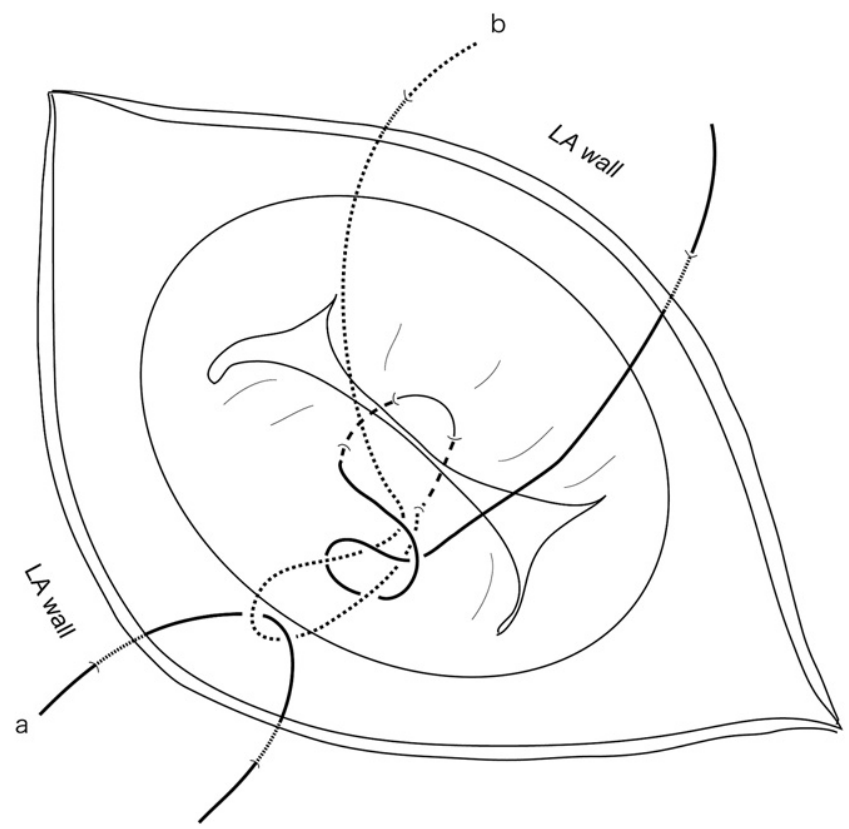

Figure 1. Temporary edge-to-edge technique model. Each string was passed through the left atrial (LA) wall. When releasing the edge-to-edge suture, string a was pulled out first, and then string $b$ was pulled out. It was not necessary to open the left atrium to remove the edge-to-edge suture. String $a$ is a 5-0 polypropylene suture, and string $b$ is a 3-0 silk suture.

investigation conforms to the "Principles of laboratory animal care" formulated by the National Society for Medical Research and the "Guide for the care and use of laboratory animals" published by the National Institute of Health and revised in 1996.

\section{Working Heart Apparatus}

The details of the working heart apparatus was described in a previous report. ${ }^{9,10}$ It is designed to perfuse the isolated heart either in a working heart mode or in Langendorff mode. The afterload column was placed $100 \mathrm{~cm}$ higher than the heart to maintain a constant afterload in the working heart mode.

\section{Instrumentation}

A high-speed digital video camera (Fastcam-PCI; Photron, Inc, Tokyo, Japan) was connected to a 10-mm-diameter rigid endoscope (Olympus Corp, Tokyo, Japan). The images were recorded at 250 fps (4-ms increment) with $512 \times 480$ resolution. Sonomicrometry (10Mz; Sonometrics Corp, London, Ontario, Canada) was used to continuously measure the distance between the probes.

\section{Animal Preparation}

Ten Landrace and Yorkshire swine weighing about $40 \mathrm{~kg}$ were used for this study. The procedure regarding the extraction of the swine heart has been previously described. ${ }^{9,10}$

A temporary EtoE suture was created in the middle portion of the leaflet edge. For protocol 1, sonomicrometric crystals were placed at the midportion of the anterior and posterior mitral valve annulus. Then the isolated heart was connected to the perfusion system. 
A 5F micromanometer-tipped catheter was introduced to the left ventricle through the apex, to the aorta, and to the left atrium through the appendage. A tube for preloading was positioned in the left atrium.

After all cannulae were applied, the heart was reperfused for the Langendorff mode. After steady cardiac beats were confirmed, the perfusion mode was changed to the working heart mode. The heart was paced at 100 beats/min with AAI pacing, and LAP was controlled from 10 to $13 \mathrm{~mm} \mathrm{Hg}$. In protocol 2 a $7 \mathrm{~F}$ conductance catheter was introduced to the left ventricle.

\section{Data Analysis}

Protocol 1. The recorded images were analyzed every $4 \mathrm{~ms}$. The timing of the motion of the mitral and aortic valves was counted at every 4 ms during 10 consecutive cardiac cycles. The average was recorded as the representative value of each run.

One cardiac cycle was divided into 4 phases: mitral valve open phase (MVOP), isovolumic contraction phase, aortic valve open phase, and isovolumic relaxation phase. MVOP was divided into the following 3 intervals to clarify which interval of MVOP changed: M1, the beginning of the mitral valve decoaptation to the time the valve was fully open; M2, fully open; And M3, the beginning of mitral valve close to the time of completely close.

The isolated hearts were paced at 100 beats/min with AAI pacing. Therefore the time of 1 cardiac cycle should be $600 \mathrm{~ms}$ in all cases, but the counted times actually ranged from $580 \mathrm{~ms}$ to 607 $\mathrm{ms}$. The ratios for one cardiac cycle of each phase were calculated, and the ratios after performing EtoE repair were compared with those after removing the EtoE suture to correct the effects of this margin of error.

The MVA was measured before and after removing the EtoE suture. The MVA of the EtoE repair was the sum of the 2 orifices. The images of the mitral valve at the full opening were obtained before and after removing the EtoE suture. The mitral effective orifice area was traced and measured in these images. The ratio of the MVA was calculated by using the following formula: Ratio of $M V A=$ MVA $($ EtoE $) / M V A(N M) \times 100(\%)$.

The changes in the anteroposterior dimension of the annular diameter were measured by means of sonomicrometry. The rate of change $(\Delta)$ was calculated as follows: $\Delta=(D L-D S) / D L$, where DL is defined as the average of 5 of the maximum distances in each cardiac cycle and DS is defined as the average of 5 of the minimum distances in each cardiac cycle.

Protocol 2. The averages of the recorded stroke volume (SV) in 5 consecutive cardiac cycles were calculated. The recorded data of the ventricular volume were differentiated by time, and these differentiated values were defined as $\mathrm{dv} / \mathrm{dt}$. The averages of the maximum $\mathrm{dv} / \mathrm{dt}$ value in each cardiac cycle were calculated, and the averages of the EtoE values were compared with those of the NM values.

\section{Statistical Analysis}

All data are summarized as the mean \pm standard deviation. Differences between the 2 groups were analyzed by means of a paired $t$ test.

\section{Results}

\section{Mitral Valve Motion}

The mitral valve motion after performing EtoE repair is shown in video images available online. The mitral valve was divided into 2 orifices of almost the same size by means of the EtoE technique. The valve of each orifice has 3 valve leaflets. The coaptation of each orifice was sufficient. The valve leaflet motion was faster and more compact than that of a normal mitral valve.

The ratios of each phase in one cardiac cycle were summarized in Figure 2. The ratios of the MVOP increased after removing the EtoE suture. The ratios of each phase were varied among each case. However, the ratios of the MVOP significantly increased after removing the EtoE suture (EtoE, 31.9\% $\pm 3.4 \%$; NM, $41.4 \pm 3.7 \% ; P=.04)$. The ratios of M1 significantly increased in the NM group (EtoE, $9.7 \% \pm 2.2 \%$; $\mathrm{NM}, 14.2 \% \pm 1.5 \% ; P=.04)$. The ratios of M2 also increased in the NM group, although they were not significantly different (EtoE, 9.1\% $\pm 3.4 \%$; NM, $15.8 \pm 2.9 \%$; $P=.08)$. M2 ratios decreased the most of the 3 parts of the MVOP (Table 1).

MVA ratios are shown in Table 2. The ratios ranged from $66.2 \%$ to $72.0 \%$, and the average was $69.6 \%$. EtoE repair reduced about $30 \%$ of the MVA and induced slight MS.

\section{Changes of the Anteroposterior Dimension of Annular Diameter}

The maximum and minimum anteroposterior diameters of the mitral valve annulus and its changes were measured by using the sonomicrometric system in 5 swine hearts. We observed no significant differences in the diameter and the changes of the anteroposterior dimensions of the mitral valve annulus after releasing the EtoE suture (maximum diameter: EtoE, $23.8 \mathrm{~mm} \pm 1.20 \mathrm{~mm}$; NM, $23.9 \mathrm{~mm} \pm 0.89 \mathrm{~mm}$, $P=.24 \mathrm{~mm}$; minimum diameter: EtoE, $20.7 \mathrm{~mm} \pm 0.8$ $\mathrm{mm}$; NM, $20.8 \mathrm{~mm} \pm 0.8 \mathrm{~mm} ; P=.92 \mathrm{~mm}$; changes: EtoE, $0.142 \mathrm{~mm} \pm 0.012 \mathrm{~mm}$; NM, $0.133 \mathrm{~mm} \pm 0.007$ $\mathrm{mm} ; P=.42 \mathrm{~mm}$; Figure 3).

\section{Volumetric Measurement}

Protocol 2 showed the SV and dv/dt changes. The dp/dt value was also measured simultaneously. The hemodynamic data for all of the hearts in the EtoE and NM groups are summarized in Table 3.

The LAP, maximum LV pressure, and $\mathrm{dp} / \mathrm{dt}$ values measured simultaneously showed no significant changes after removing the EtoE suture. Both the peak positive and peak negative $\mathrm{dp} / \mathrm{dt}$ values showed no obvious change. The dp/dt represents the ability of the heart to contract and relax.

Therefore both the systolic and diastolic LV function revealed no significant changes by performing the EtoE repair. The SV also showed no significant changes after removing the EtoE suture (EtoE, $11.1 \pm 0.7 \mathrm{~mL}$; NM, $10.7 \pm 0.6$ $\mathrm{mL} ; P=.23)$. The $\mathrm{dv} / \mathrm{dt}$ increased slightly after removing the EtoE sutures, but the difference was not significant $($ EtoE, $118.4 \pm 25.4 \mathrm{~mL} / \mathrm{s} ; \mathrm{NM}, 130.0 \pm 17.2 \mathrm{~mL} / \mathrm{s}$; $P=.14)$. 


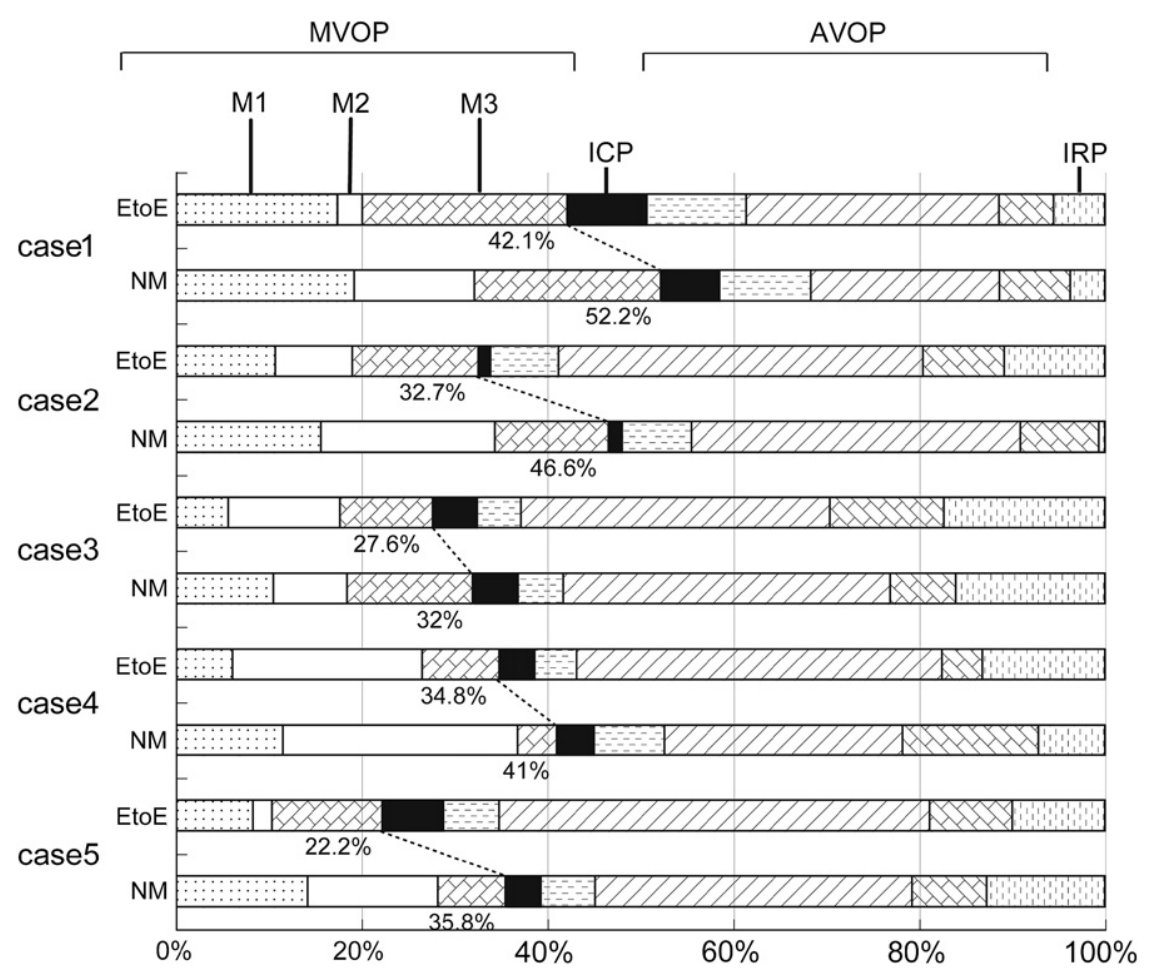

Figure 2. The ratios of each phase in one cardiac cycle. The written percentage beside the bar is the ratio of the mitral valve open phase. EtoE, Edge-to-edge repair; NM, normal mode; MVOP, mitral valve open phase; AVOP, aortic valve open phase; $I C P$, isovolumic contraction phase; IRP, isovolumic relaxation phase.

\section{Discussion}

The present study evaluated the mitral valve motion after performing EtoE repair with a $U$ stitch and compared it with the normal mitral valve in the swine working heart model by using a high-speed digital video camera system. In addition, a temporary EtoE model was created by using a removable Alfieri suture. This model reveals the EtoE conditions first and is changed to a normal mitral valve condition easily by removing the Alfieri suture, thus allowing for the examination of the mitral valve and heart function in the both the EtoE and NM groups within a short time period without any damage to cardiac function. The conversion from EtoE repair to $\mathrm{NM}$ simply requires pulling out the tie in a bow on the mitral valve, and there is no adverse affect on heart function (see online video).
The ratio of each cardiac phase in a cardiac cycle was evaluated. The echocardiographic assessment might also provide reliable results. However, evaluation with a high-speed video camera was more precise in time-related analysis of the valve motion because the images were recorded at high frame $(250$ fps). MVOP ratios were significantly decreased by using the EtoE technique. The ratio of M1 was remarkably decreased, and the M2 ratio was also decreased. The M2 ratio was decreased most of the 3 periods of the MVOP.

The cause of this change is not clear. The EtoE technique divided the mitral orifice and created a double orifice. Each orifice is smaller than the original mitral orifice. It allows for the easy and quick opening of the mitral valve. This suggests that the reason for shortening the MVOP by using the EtoE procedure is the small size of the double orifice of the

TABLE 1. The changes of 3 periods of the MVOP

\begin{tabular}{|c|c|c|c|c|c|c|}
\hline & \multicolumn{2}{|c|}{ M1 } & \multicolumn{2}{|c|}{ M2 } & \multicolumn{2}{|c|}{ M3 } \\
\hline & EtoE & NM & EtoE & NM & EtoE & NM \\
\hline Case $1, \mathrm{~ms}(\%)$ & $105(17.5)$ & $115(19.1)$ & $16(2.7)$ & $78(13.0)$ & $132(22.0)$ & $121(20.1)$ \\
\hline Case 2 & $66(10.9)$ & $91(15.7)$ & $50(8.2)$ & $108(18.6)$ & $82(13.5)$ & $71(12.2)$ \\
\hline Case 3 & $34(5.6)$ & $64(10.5)$ & $73(12.0)$ & $48(7.9)$ & $60(9.9)$ & $82(13.5)$ \\
\hline Case 4 & $37(6.2)$ & $70(11.6)$ & $122(20.4)$ & $153(25.3)$ & $50(8.3)$ & $25(4.1)$ \\
\hline Case 5 & $51(8.4)$ & $86(14.2)$ & $12(2.0)$ & $85(14.0)$ & $72(11.9)$ & $44(7.3)$ \\
\hline \multirow[t]{2}{*}{ Average (\%) } & $9.7 \pm 2.2$ & $14.2 \pm 1.5$ & $9.1 \pm 3.4$ & $15.8 \pm 2.9$ & $13.1 \pm 2.4$ & $11.4 \pm 2.7$ \\
\hline & \multicolumn{2}{|c|}{$P=.04$} & \multicolumn{2}{|c|}{$P=.08$} & \multicolumn{2}{|c|}{$P=.23$} \\
\hline
\end{tabular}

MVOP, Mitral valve open phase; EtoE, edge-to-edge repair; NM, normal mode. 
TABLE 2. The ratio of the mitral valve area of 5 swine hearts

Ratio of MVA (EtoE/NM)

\begin{tabular}{lc}
\hline Case 1 & $69.2 \%$ \\
Case 2 & $70.0 \%$ \\
Case 3 & $70.4 \%$ \\
Case 4 & $72.0 \%$ \\
Case 5 & $66.2 \%$ \\
Average & $69.6 \% \pm 0.02 \%$ \\
\hline
\end{tabular}

MVA, Mitral valve area; EtoE, edge-to-edge repair; $N M$, normal mode.

mitral valve. The mechanism regarding the M2 decrease was not clarified. The video clip (videoclip) shows that the anterior and posterior leaflet was sutured by using the EtoE technique, which pulled both leaflets in a manner similar to pulling sails. During M2, the EtoE technique divides the orifice into 2 small orifices, and both leaflets were retracted by the EtoE technique. The EtoE technique makes the leaflets small, thus producing a large amount of traction on the leaflets. The small leaflets might therefore restrict the valve's ability to open, and such traction might make the leaflets close too early.

MVA after EtoE repair is still a major concern for cardiac surgeons. Previous reports using echocardiography have shown that the EtoE repair did not create MS; however, there are no reports describing the direct observation of the mitral valve after EtoE repair. In the current study we directly observed mitral valve motion before and after the EtoE technique.

In this study the MVA was decreased by about $30 \%$ after performing the EtoE technique. Jimenez and colleagues ${ }^{11}$ demonstrated that EtoE repair alone decreased the effective orifice area by approximately $16 \%$. Our results showed slightly greater decrease than their observation. We observed the mitral valve through the LV apex and took a nearly frontal view of it and calculated the MVA using a tracing method. The mitral valve view was not a right frontal view, and it pro- vided only 2-dimensional information without depth. This mode of observation might underestimate the MVA. However, the MVA after performing the EtoE technique maintained at least $70 \%$ of the normal MVA, and the MVA decrease of this magnitude did not cause functional MS.

In addition, MS after performing the EtoE technique was evaluated from the viewpoint of the SV and the dv/dt. The dv/ $\mathrm{dt}$ has not been previously used to evaluate MS, whereas the negative $\mathrm{dv} / \mathrm{dt}$ has been used to evaluate the cardiac output in one report. ${ }^{12}$ In unpublished data from a previous study, the $\mathrm{dv} / \mathrm{dt}$ and the SV showed no significant changes when creating mild MS and decreased significantly only when critical MS was created.

In this study the SV did not change before or after performing the EtoE technique. It indicated that the inflow volume of the left ventricle thus did not change because of the EtoE technique. The dv/dt showed a tendency to decrease after the EtoE technique but without significance. These results indicated that the EtoE technique does not create mitral obstruction. This observation supports the echocardiographic findings reported in previous reports. $4,5,7,8$

Our study shows that the EtoE technique decreases the MVOP by $10 \%$ and the MVA by $30 \%$. In addition, the rate of LV filling $(\mathrm{dv} / \mathrm{dt})$ was observed to be lower after the EtoE technique, although there is no significant difference. However, the SV was unchanged. These observations might suggest that the left ventricle was ejecting at a smaller end-diastolic volume, and the ejection fraction also increased. One the other hand, in this study the end-diastolic volume showed no significant difference $(P=.35)$ and the ejection fraction also showed no significant difference, although there was a tendency for this value to increase in the EtoE group $(P=.10)$.

Mitral annular contraction coincident with mitral valve motion has been reported. ${ }^{13-15}$ The effect of the EtoE technique on mitral annular contraction was evaluated, but there
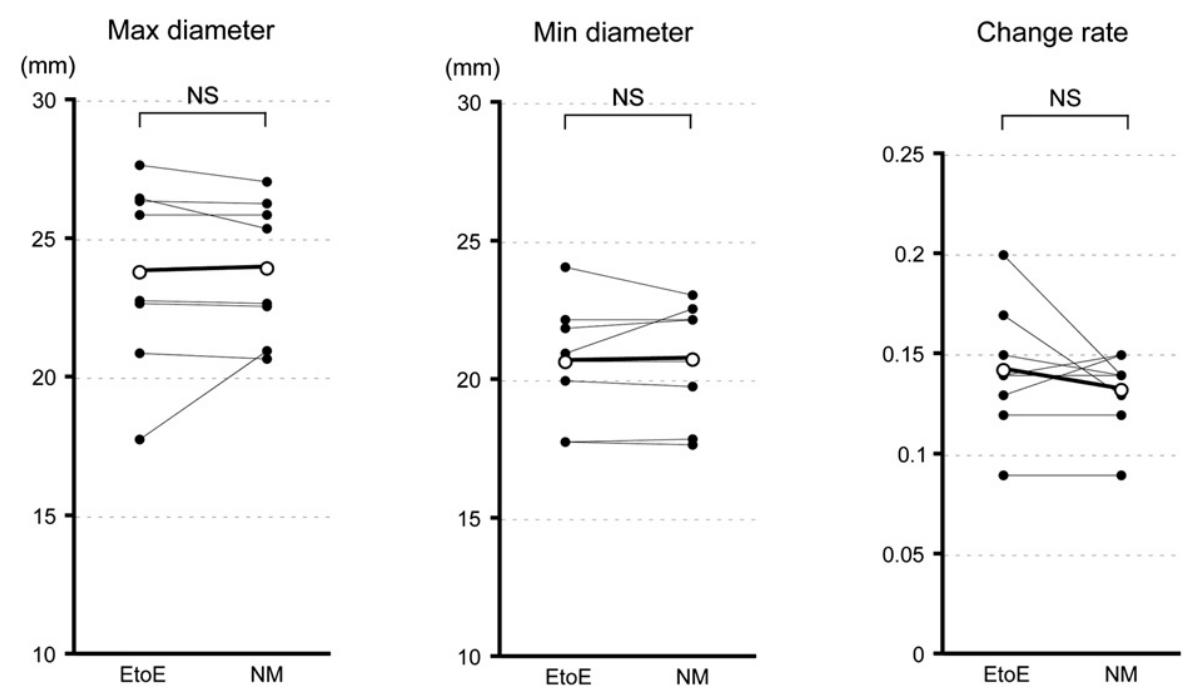

Figure 3. Changes in the anteroposterior dimension of the annular diameter. There was no significant difference in the diameter and the changes of anteroposterior dimension of the mitral annulus after releasing the edge-to-edge suture. EtoE, Edge-to-edge repair; NM, normal mode. 
TABLE 3. Hemodynamic data on the EtoE and the NM

\begin{tabular}{|c|c|c|c|c|c|c|c|c|}
\hline & & $\begin{array}{c}\text { LAP } \\
(\mathrm{mm} \mathrm{Hg})\end{array}$ & $\begin{array}{l}\text { Maximum } \\
\text { LVP (mm Hg) }\end{array}$ & $\begin{array}{c}\text { Peak positive } \\
\text { dp/dt (mm Hg/s) }\end{array}$ & $\begin{array}{c}\text { Peak negative } \\
\text { dp/dt (mm Hg/s) }\end{array}$ & $\begin{array}{c}\text { Stroke } \\
\text { volume }(\mathrm{mL})\end{array}$ & $\begin{array}{c}\text { Peak positive } \\
\text { dv/dt (mL/s) }\end{array}$ & $\begin{array}{c}\text { Peak negative } \\
\text { dv/dt }(\mathrm{mL} / \mathrm{s})\end{array}$ \\
\hline \multirow[t]{2}{*}{ Case 1} & EtoE & 12 & $78.5 \pm 0.2$ & $587 \pm 6.7$ & $-435 \pm 3.5$ & $11.1 \pm 0.2$ & $126.0 \pm 3.0$ & $-68.8 \pm 4.0$ \\
\hline & NM & 10 & $79.8 \pm 0.1$ & $592 \pm 2.7$ & $-533 \pm 2.7$ & $11.1 \pm 0.2$ & $144.4 \pm 8.3$ & $-64.0 \pm 2.2$ \\
\hline \multirow[t]{2}{*}{ Case 2} & EtoE & 8 & $82.4 \pm 1.9$ & $636 \pm 16.7$ & $-532 \pm 17.9$ & $11.8 \pm 0.6$ & $144.0 \pm 2.2$ & $-63.0 \pm 2.7$ \\
\hline & NM & 9 & $85.9 \pm 9.2$ & $656 \pm 29.7$ & $-544 \pm 29.7$ & $10.6 \pm 1.3$ & $132.0 \pm 11$ & $-63.0 \pm 4.5$ \\
\hline \multirow[t]{2}{*}{ Case 3} & EtoE & 11 & $84.1 \pm 0.1$ & $648 \pm 11.0$ & $-548 \pm 26.8$ & $10.5 \pm 0$ & $93.0 \pm 2.7$ & $-77.0 \pm 2.7$ \\
\hline & NM & 11 & $83.2 \pm 0.2$ & $660 \pm 0$ & $-540 \pm 0$ & $10.1 \pm 0.3$ & $112.0 \pm 2.7$ & $-85.0 \pm 0$ \\
\hline \multirow[t]{2}{*}{ Case 4} & EtoE & 16 & $86.1 \pm 0.1$ & $608 \pm 11.0$ & $-520 \pm 0$ & $10.2 \pm 0.1$ & $90.0 \pm 0$ & $-77.0 \pm 2.7$ \\
\hline & NM & 16 & $83.7 \pm 0.1$ & $620 \pm 0$ & $-480 \pm 0$ & $10.3 \pm 0$ & $113.0 \pm 2.7$ & $-85.0 \pm 0$ \\
\hline \multirow[t]{2}{*}{ Case 5} & EtoE & 11 & $89.8 \pm 0.1$ & $586 \pm 8.2$ & $-653 \pm 7.6$ & $11.8 \pm 0.3$ & $138.8 \pm 2.8$ & $-70.4 \pm 1.8$ \\
\hline & NM & 11 & $89.5 \pm 0.2$ & $584 \pm 9.6$ & $-650 \pm 7.1$ & $11.5 \pm 0.2$ & $148.8 \pm 2.5$ & $-69.2 \pm 1.1$ \\
\hline \multirow[t]{2}{*}{ Average } & EtoE & $11.6 \pm 2.9$ & $84.2 \pm 4.2$ & $613.0 \pm 28.2$ & $-537.6 \pm 77.9$ & $11.1 \pm 0.7$ & $118.4 \pm 25.4$ & $-70.8 \pm 6.2$ \\
\hline & NM & $\begin{array}{c}11.4 \pm 2.7 \\
\mathrm{NS}\end{array}$ & $\begin{array}{c}84.4 \pm 3.6 \\
\text { NS }\end{array}$ & $\begin{array}{c}622.4 \pm 35.2 \\
\text { NS }\end{array}$ & $\begin{array}{c}-549.4 \pm 61.9 \\
\text { NS }\end{array}$ & $\begin{array}{c}10.7 \pm 0.6 \\
\mathrm{NS}\end{array}$ & $\begin{array}{c}130.0 \pm 17.2 \\
\text { NS }\end{array}$ & $\begin{array}{c}-73.2 \pm 10.9 \\
\text { NS }\end{array}$ \\
\hline
\end{tabular}

EtoE, Edge-to-edge repair; $N M$, normal mode; $L A P$, left atrial pressure; $L V P$, left ventricular pressure; $N S$, not significant.

were no significant differences in the distance and change rate of the anteroposterior dimension of annular diameter before or after removing the EtoE suture. Therefore the EtoE technique has no adverse affect on the mitral annular dynamics.

In summary, the EtoE technique using one stitch had a low risk of creating MS and did not affect the cardiac output, although the EtoE technique slightly affected the inflow volume of the ventricle. Under clinical circumstances, concomitant annuloplasty or additional valve repair techniques might therefore reduce the MVA. The diseased valves often become thicker, and these valves might thereafter further decrease the MVOP. Atrial fibrillation without atrial kick also decreases the MVOP. These situations together lead to a decrease in the $\mathrm{dv} / \mathrm{dt}$ value, thus resulting in adverse affects on the cardiac function.

\section{Limitations}

First, the present study is designed in a swine working heart model, and it does not fully resemble a human heart in a living body. The heart is circulated with a crystalloid perfusion, the pulmonary vasculature is eliminated, and the geometry of the heart is not physiologic.

Second, a temporary EtoE model was created that achieves a quick conversion from the EtoE technique to the NM without any functional damage to the heart. However, the EtoE technique is first and the NM is second. This swine working heart model maintains a steady and reliable function over 4 hours; however, the order used in this study might have had an adverse affect on the results.

Third, the function of a normal swine heart was examined. It is different from a diseased heart with mitral regurgitation. The mitral valve motion was also examined on the normal valve, which might be different from the diseased one. Under clinical circumstances, more aggressive EtoE repair, con- comitant annuloplasty, or other valve repair procedures might therefore be required because of the redundant and degenerative valve or the dilated annulus. The results indicated in this study might be different from those in the diseased heart when the EtoE technique is applied clinically. MVA might thus become smaller, and MS might therefore be observed, depending on the situation.

\section{Conclusions}

A temporary EtoE model was created to estimate the motion of mitral valve after EtoE repair by using a high-speed video camera system in an isolated swine heart. Each orifice has 3 valve leaflets and had satisfactory coaptation. The MVOP decreased after performing the EtoE technique. The EtoE technique did not affect the mitral annular contraction.

The MVA decreased by approximately $30 \%$ after performing the EtoE technique. The SV does not change, and the $\mathrm{dv} / \mathrm{dt}$ value decreased slightly without significance after performing the EtoE technique. However, the EtoE technique did not create symptomatic MS and showed no adverse affects on the cardiac function.

We thank Professor Nobuyuki Hamajima of Nagoya University Graduate School of Medicine for his valuable advice on statistical analysis and also Akira Oonishi, $\mathrm{PhD}$, of Gene Research Center University of Tsukuba for his supply of animals used in this study.

\section{References}

1. Fucci C, Sandrelli L, Pardini A, Torracca L, Ferrari M, Alfieri O. Improved results with mitral valve repair using new surgical techniques. Eur J Cardiothorac Surg. 1995;9:621-7.

2. Maisano F, Torracca L, Oppizzi M, Stefano PL, D'Addario G, La Canna G, et al. The edge-to-edge technique: a simplified method to correct mitral insufficiency. Eur J Cardiothorac Surg. 1998;13:240-6.

3. Maisano F, Schreuder JJ, Oppizzi M, Fiorani B, Fino C, Alfieri O. The double-orifice technique as a standardized approach to treat mitral regurgitation due to severe myxomatous disease: surgical technique. Eur $J$ Cardiothorac Surg. 2000;17:201-5. 
4. Bhudia SK, McCarthy PM, Smedira NG, Lam BK, Rajeswaran J, Blackstone EH. Edge-to-edge (Alfieri) mitral repair: results in diverse clinical settings. Ann Thorac Surg. 2004;77:1598-606.

5. Brinster DR, Unic D, D'Ambra MN, Nathan N, Cohn LH. Midterm results of the edge-to-edge technique for complex mitral valve repair. Ann Thorac Surg. 2006;81:1612-7.

6. Alfieri O, Maisano F, De Bonis M, Stefano PL, Torracca L, Oppizzi M, et al. The double-orifice technique in mitral valve repair: a simple solution for complex problems. J Thorac Cardiovasc Surg. 2001;122: 674-81.

7. Timek TA, Nielsen SL, Liang D, Lai DT, Dagum P, Daughters GT, et al. Edge-to-edge mitral repair: gradients and three-dimensional annular dynamics in vivo during inotropic stimulation. Eur J Cardiothorac Surg. 2001;19:431-7.

8. Kinnaird TD, Munt BI, Ignaszewski AP, Abel JG, Thompson RC. Edgeto-edge repair for functional mitral regurgitation: an echocardiographic study of the hemodynamic consequences. J Heart Valve Dis. 2003;12: 280-6.

9. Araki Y, Usui A, Kawaguchi O, Saito S, Song MH, Akita T, et al. Pressure-volume relationship in isolated working heart with crystalloid per- fusate in swine and imaging the valve motion. Eur J Cardiothorac Surg. 2005;28:435-42.

10. Saito S, Araki Y, Usui A, Akita T, Oshima H, Yokote J, et al. Mitral valve motion assessed by high-speed video camera in isolated swine heart. Eur J Cardiothorac Surg. 2006;30:584-91.

11. Jimenez JH, Forbess J, Croft LR, Small L, He Z, Yoganathan AP. Effects of annular size, transmitral pressure, and mitral flow rate on the edge-toedge repair: an in vitro study. Ann Thorac Surg. 2006;82:1362-8.

12. Al-Shafei AI, Wise RG, Gresham GA, Carpenter TA, Hall LD, Huang CL. Magnetic resonance imaging analysis of cardiac cycle events in diabetic rats: the effect of angiotensin-converting enzyme inhibition. J Physiol. 2002;538(suppl):555-72.

13. Glasson JR, Komeda M, Daughters GT, Foppiano LE, Bolger AF, Tye TL, et al. Most ovine mitral annular three-dimensional size reduction occurs before ventricular systole and is abolished with ventricular pacing. Circulation. 1997;96(suppl):II115-23.

14. Timek T, Dagum P, Lai DT, Green GR, Glasson JR, Daughters GT, et al. The role of atrial contraction in mitral valve closure. J Heart Valve Dis. 2001;10:312-9.

15. Timek TA, Green GR, Tibayan FA, Lai DT, Rodriguez F, Liang D, et al. Aorto-mitral annular dynamics. Ann Thorac Surg. 2003;76:1944-50.

\section{The Journal of Thoracic and Cardiovascular Surgery Conflict of Interest Policy}

To assure fairness to authors submitting work for consideration in The Journal of Thoracic and Cardiovascular Surgery, a mechanism exists for managing conflicts of interest. The editor and each of the section editors complete a "Conflict of Interest" form that identifies any and all relationships with commercial and other academic entities. When the editor has a potential conflict because of a relationship with another entity or author, the editor appoints an alternate editor from among the section editors or editorial board members who assumes the entire responsibility for final decisions on the manuscript in question. The editor does not read the reviews that are submitted nor engage in discussing the manuscript prior to the final decision. When the conflict of interest involves a section editor, a "guest section editor" is appointed who fills the role normally played by the conflicted section editor. All members of the editorial board and reviewers are asked to indicate any conflict of interest when they agree to review a manuscript. 